\title{
Quantitative Proteomic Analysis Shows Involvement of the p38 MAPK Pathway in Bovine Parainfluenza Virus Type 3 Replication
}

\section{Liyang Li}

Heilongjiang Bayi Agricultural University https://orcid.org/0000-0002-4633-9419

\section{Pengfei Li}

The Fifth Affiliated Hospital of Harbin Medical University

\section{Ao Chen}

Heilongjiang Bayi Nongken University: Heilongjiang Bayi Agricultural University

Hanbing Li

Heilongjiang Bayi Agricultural University

\section{Zhe Liu}

Heilongjiang Bayi Agricultural University

\section{Liyun Yu}

Heilongjiang Bayi Agricultural University

\section{Xilin Hou ( $\nabla$ xly_hou@163.com )}

Heilongjiang Bayi Agricultural University

\section{Research Article}

Keywords: Bovine parainfluenza virus type 3 (BPIV3), Differentially expressed proteins, p38 MAPK signaling pathway, Quantitative proteomics

Posted Date: February 26th, 2021

DOl: https://doi.org/10.21203/rs.3.rs-253558/v1

License: (c) (1) This work is licensed under a Creative Commons Attribution 4.0 International License. Read Full License 
1 Quantitative proteomic analysis shows involvement of the p38

2 MAPK pathway in bovine parainfluenza virus type 3 replication

3 Liyang $\mathrm{Li}^{\text {a, b,1 }}$, Pengfei Lic, ${ }^{\mathrm{c}, 1}$, Ao Chen ${ }^{\mathrm{a}}$, Hanbing $\mathrm{Li}^{\mathrm{a}}$, Zhe Liu ${ }^{\mathrm{a}}$, Liyun Yu ${ }^{\mathrm{a}^{*}}$, Xilin Hou ${ }^{\text {b* }}$

4 a College of Life Science and Biotechnology, Heilongjiang Bayi Agricultural University, Daqing

5 163319, China

6 b College of Animal Science and Veterinary Medicine, Heilongjiang Bayi Agricultural University,

7 Daqing 163319, China

8 c Department of Nephrology, The Fifth Affiliated Hospital of Harbin Medical University, Daqing

9 163319, China

1 These authors equally contributed to this work.

11

* Corresponding author. Tel.: +86 04596819291 ; fax: +86 04596819291.

E-mail address: xly_hou@163.com,yuliyun1227@126.com

13

14 


\section{Abstract}

Bovine parainfluenza virus type 3 (BPIV3) infection often causes respiratory tissue damage and immunosuppression and results in bovine respiratory disease complex. Bovine respiratory disease complex is one of the major diseases in dairy cattle and causes huge economical losses every year. The pathogenetic and immunoregulatory mechanisms involved in the process of BPIV3 infection, however, remain unknown. Proteomics is a powerful tool for high-throughput identification of proteins and has been widely used to understand how viruses interact with host cells. In the present study, we report a proteomic analysis to investigate the whole cellular protein alterations of MDBK cells infected with BPIV3. To investigate the invasion process of BPIV3 and the immune response mechanism of MDBK cells, isobaric tags for relative and absolute quantitation analysis (iTRAQ) and Q-Exactive mass spectrometry-based proteomics were performed. The differentially expressed proteins (DEPs) involved in the BPIV3 invasion process in MDBK cells were identified, annotated, and quantitated. A total of 116 proteins, which included 74 upregulated proteins and 42 downregulated proteins, were identified as DEPs between the BPIV3-infected and the mock-infected groups. These DEPs included corresponding proteins related to inflammatory response, immune response, and lipid metabolism. These results might provide some insights for understanding the pathogenesis of BPIV3. Fluorescent quantitative PCR and western blotting analysis showed results consistent with those of iTRAQ identification. Interestingly, the upregulated protein MKK3 was associated with the p38 MAPK signaling pathway. The results of proteomics analysis indicated BPIV3 infection could activate the p38 MAPK pathway to promote virus replication.

Keywords: Bovine parainfluenza virus type 3 (BPIV3); Differentially expressed proteins; p38 MAPK signaling pathway; Quantitative proteomics

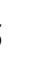

6

.

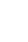

0

1




\section{Introduction}

Bovine parainfluenza virus type 3 (BPIV3) is an enveloped, single-stranded negative-sense RNA virus that belongs to the family Paramyxoviridae, genus Respirovirus[1]. BPIV3 infection results in pneumonia and atypical interstitial pneumonia in cattle and leads to severe secondary bacterial infection and other related clinical symptoms. BPIV3 infection and other viral or bacterial infections often cause bovine respiratory disease complex (BRDC)[2]. The mortality of cattle due to BRDC is up to $35 \%$, which causes huge economic losses in the cattle industry[3] . The genome of BPIV3 contains a single-stranded negative-sense RNA of approximately $15 \mathrm{~kb}$ in size and encodes six structural proteins and three nonstructural proteins [4-6]. The structural proteins of BPIV3 include nucleoprotein $(\mathrm{N})$, phosphoprotein $(\mathrm{P})$, large protein $(\mathrm{L})$, matrix protein (M), hemagglutinin-neuraminidase $(\mathrm{HN})$, and the homotrimeric fusion $(\mathrm{F})$, while the accessory nonstructural proteins include $\mathrm{C}, \mathrm{V}$, and $\mathrm{D}$ proteins. Multiple functions and activities of the structural and accessory proteins have been investigated. For example, the glycoprotein $\mathrm{HN}$ binds to the receptor protein on the host cell surface and mediates membrane fusion[7-8]. The $\mathrm{M}$ protein is located in the underlayer of the envelope, and is essential in the assembly, replication, and release processes of virus particles. The nonstructural proteins including $\mathrm{V}$ protein and $\mathrm{C}$ protein are also encoded by the $\mathrm{P}$ gene. The $\mathrm{V}, \mathrm{C}$, and $\mathrm{N}$ proteins together regulate virus replication [9]. Although much progress has been made in understanding the proteins of BPIV3, the pathogenetic and immunoregulatory mechanisms involved in the process of BPIV3 infection remain largely unclear. To investigate the changes in the host physiological system during the process of viral invasion, isobaric tags for relative and absolute quantitation analysis (iTRAQ) mass spectrometry (MS)-based global proteomics profiling was performed.

The iTRAQ quantitative proteomics technique has been widely used to study interaction between virus and host based on high sensitivity and quantitation accuracy[10-11]. An et al. used iTRAQ to determine the differentially expressed proteins (DEPs) of transmissible gastroenteritis virus (TGEV)-infected PK-15 cells. The authors identified 60 upregulated and 102 downregulated proteins in the TGEV infection process. Their analysis revealed that many upregulated proteins were associated with interferon signaling and that TGEV infection could activate the JAK-STAT1 signaling pathway[12]. Sun et al. used the iTRAQ quantitative proteomics technique to identify proteins associated with porcine epidemic diarrhea virus (PEDV) infection in order to provide a scientific basis for the pathogenesis of PEDV [13]. Presently, iTRAQ had become the main quantitative proteomics technology to understand the process of viral infection. In the present study, the DEPs in BPIV3-infected MDBK cells were identified and quantitatively analyzed by using an iTRAQ-based proteomics approach. MDBK cells have been selected for use in many studies[14-15]. MDBK cells are commonly used not only for BPIV3 isolation, propagation and basic research, but also as hosts for many different bovine pathogens, including bovine respiratory syncytial virus (BRSV) and bovine 
herpesvirus type 1 [16-17].

The expression levels of 116 proteins were found to be significantly altered after $24 \mathrm{~h}$ of BPIV3 infection. These cellular DEPs were assigned to several biological processes according to bioinformatics analysis. The results of this study provide a global understanding of the host's immune response to BPIV3 infection.

\section{Materials and methods}

\section{Virus infection of MDBK cells}

MDBK cells were cultured in DMEM (Dulbecco's modified Eagle's medium) medium containing $10 \%$ fetal bovine serum (FBS) and $100 \mathrm{~g} / \mathrm{ml}$ penicillin and $100 \mathrm{~g} / \mathrm{ml}$ streptomycin. Cell culture conditions at $37{ }^{\circ} \mathrm{C}$ with $5 \% \mathrm{CO}_{2}$ in $24 \mathrm{~h}$. The BPIV3 DQ strain (GenBank accession no. HQ462571) was isolated and identified in the preventive veterinary laboratory of Heilongiang Bayi Agricultural University. MDBK cells were transduction with BPIV3 at multiplicity of infection $(\mathrm{MOI}=1)$. Uninfected cells were used as mock-infected groups. Each experiment were carried out with three replicates. The cytopathic effect (CPE)was observed and the growth curve of BPIV3 was measured. TCID 50 were measured by the Reed-Muench method.

\section{Protein isolation, digestion, and labeling with iTRAQ reagents}

All the cells samples, included BPIV3-infected group and control group, were cleaned with cold PBS twice and centrifuged at $1000 \mathrm{~g}$ at $4{ }^{\circ} \mathrm{C}$ for 10 minutes to harvested cells. Then, collected cells were lysed to extract proteins in the $300 \mu$ SDT ( 1 mM PMSF, 2 mM EDTA and 10 $\mathrm{mM}$ DTT). Protein samples of dissolved were harvested with centrifugation at $14000 \mathrm{~g}$ for $40 \mathrm{~min}$ at $4{ }^{\circ} \mathrm{C}$. The concentration of harvested protein supernatant was determined using BCA protein assay. Results showed that $100 \mu \mathrm{g}$ protein was digested for $8 \mathrm{~h}$ at $37{ }^{\circ} \mathrm{C}$ using sequencing-grade modified trypsin. The protein samples were labeled by different iTRAQ tags on the basis of iTRAQ Reagent-8plex Multiplex Kit instruction (AB SCIEX). Three mock-infected samples were labeled by iTRAQ 113, iTRAQ 114, and iTRAQ 115, respectively; three BPIV3-infected samples were labeled by iTRAQ 116, iTRAQ 117 and iTRAQ 118, respectively. Then the labeled samples were mixed and dried by using vacuum concentrator.

\section{LC-MS/MS Analysis}

The labeled peptide samples were purified and separated by AKTA purification system. The operation methods and solution preparation were performed essentially as described previously [18]. The whole elution process was monitored at $214 \mathrm{~nm}$ and collected every minute. Thirty distillates were collected and neutralized in 10 pools and desalinated in a 18 cartridge. After each fraction was vacuum centrifuged, the sample was dissolved in $40 \mu \mathrm{L} 0.1 \%$ trifluoroacetic acid and kept frozen at $-80{ }^{\circ} \mathrm{C}$ for mass spectrometry analysis. Each sample was separated by capillary high-performance liquid chromatography (Thermo scientific EASY column $(2 \mathrm{~cm}, 100 \mu \mathrm{m} 5 \mu \mathrm{m}$, 
$139 \mathrm{C} 18)$. The chromatography conditions were as follow: Water with $0.1 \%$ formic acid (A) and

140 Acetonitirile with $0.1 \%$ formic acid (B) were used as mobile phage. The flow rate was $300 \mathrm{~nL}$ per

141 minute and the mobile phage gradient program was used: $0-33$ minute, from $0 \%$ to $40 \%(\mathrm{~B})$;

$142 \quad 33-34$ minute, B from $40 \%$ to $100 \%$ (B); 34- 35minute maintained $100 \%$ and then back to $40 \%$.

143 Then, proteins were analyzed by using a Q-Exactive mass spectrometry (Thermo Finnigan) at

144 positive ion mode (parameters: mass range: 300-1800m/z; Dynamic exclusion: $40.0 \mathrm{~s}$, MS2

145 Activation Type: HCD, Normalized collision energy: $30 \mathrm{eV}$ ).

\section{Database search and bioinformatic analysis}

$147 \quad$ MS/MS data were searched against the bovine subset database from the UniProt database 148 (release March 22, 2016, containing 32015 sequences) and proteins were identified by using 149 Mascot 2.3.02. The peptide for quantification was automatically selected by Paragon ${ }^{\mathrm{TM}}$ algorithm 150 to calculate the reporter peak area, error factor (EF) and p-value. The proteins expression levels of 151 BPIV3-infected cells were calculated to compare with those of mock-infected cells. Proteins with 152 fold changes $>1.5$ and $p$-values $<0.05$ were considered as significantly different expressions. Auto 153 bias-corrected were executed to decrease artificial error. These proteins were further classified 154 using Gene Ontology (GO) and pathway enrichment analysis (http://www.geneontology.org).

\section{RNA extraction and real-time PCR analysis}

The mRNA level of differentially expressed proteins was analyzed by real-time PCR. Total RNA was extracted from cells of the BPIV3 infected group and the control group using TRIzol reagent(Takara) according to the manufacturer's protocol. The RNA concentration was measured using NanoDropnd-1000. After extraced, $1 \mu \mathrm{L}$ total RNA was detected using electrophoresis. The cDNAs of these samples were obtained by reverse transcription. Relative quantitative real-time PCR was performed in a $25 \mu \mathrm{L}$ system that containing $12.5 \mu \mathrm{L}$ SYBR Premix Ex TaqTM II, $2 \mu \mathrm{L}$ primers, $2 \mu \mathrm{L}$ cDNA samples and $8.5 \mu \mathrm{L}$ water. The reaction condition was $95{ }^{\circ} \mathrm{C}$ for $10 \mathrm{~min}$, then 40 cycles of $95{ }^{\circ} \mathrm{C}$ for $30 \mathrm{~s}, 57^{\circ} \mathrm{C}$ for $30 \mathrm{~s}$ and $72{ }^{\circ} \mathrm{C}$ for $30 \mathrm{~s}$, Then, the melting curves were obtained. The gene of GADPH was used as the internal reference gene. The data statistic was based on three independent experiments.

\section{Western-blot}

MDBK cells were washed two times with PBS and disrupted with lysis buffer (50 mM Tris- $\mathrm{HCl}, \mathrm{pH} 8.0,150 \mathrm{mM} \mathrm{NaCl}$ and $1 \%$ Triton $\mathrm{X}-100$, supplemented with 1 tablet of

169 Complete-Mini Protease Inhibitor Cocktail per $50 \mathrm{ml}$ buffer). Cell lysates were centrifugated at

$17012,000 \times \mathrm{g}$ for $10 \mathrm{~min}$ to harvest supernatants. Protein assays were performed on all supernatants 171 using the Bradford method. For Western blot analysis of whole-cell lysates, samples, each 172 containing $25-30 \mu \mathrm{g}$ of protein equivalent, were dissociated in SDS-PAGE loading buffer and 173 separated by $12 \%$ gradient SDS-PAGE. Proteins were then transferred to a nitrocellulose 174 membrane. After sample separation by electrophoresis, proteins were transferred to an 
175 Immobilon-FL membrane (Millipore) via electroblotting. Primary anti-bodies and dilutions

176 including MKK3 (rabbit, Cell Signal Technology5674, Danvers, MA), p38 phosphorylation 177 (p-38) at 1:1000 (mouse, Cell Signal Technology9216, Danvers, MA ), p-38 at 1:1000 (rabbit, 178 Cell Signal Technology41666, Danvers, MA), $\beta$-actin at 1:10,000 (mouse, Sigma) and were incubated with membrane at $4{ }^{\circ} \mathrm{C}$ over-night. As a secondary antibody, anti rabbit

180 immunoglobulin G (Santa Cruz Biotechnology Inc.) was applied (1:1000, rabbit or mouse) at room temperature for $1 \mathrm{~h}$. After further washes, the immune complexes were revealed by enhanced chemluminescence using the ECL detection kit (Beijing Biosea Biotechnology Co., Ltd.).

\section{Indirect immunofluorescent assay (IFA)}

The operation methods were performed essentially as described previously [14]. MDBK cells were fixed with $4 \%$ paraformaldehyde for $10 \mathrm{~min}$, permeabilized with $0.1 \%$ Trinton X-100 for $20 \mathrm{~min}$, blocked with 1\% BSA for $2 \mathrm{~h}$, and washed with PBS three times. Cells were incubated with $200 \mu \mathrm{L}$ rabbit polyclonal anti-BPIV3 serum (1:100) for $1 \mathrm{~h}$, followed by incubation with 200 $\mu \mathrm{L}$ FITC-conjugated Goat anti-rabbit IgG (1:100) for $1 \mathrm{~h}$ at room temperature. The cells were washed extensively with PBS, and 90\% glycerol was added to mount cells. Cells were examined and images were captured using fluorescence microscope.

\section{Statistical analysis}

Statistical analysis was performed in Microsoft Excel for two-tailed Student's $t$ test or one-way analysis of variance (ANOVA) and the p-values $<0.05$ were considered statistically significant.

\section{Results}

\section{Detection of the activity of BPIV3 in MDBK cells}

In proteomics analysis of virus-infected cells, the optimal sampling time is when the virus replicates quickly and the cells have no significant pathological effect (CPE). To determine the optimal sampling time point for proteomics analysis after BPIV3 infection, MDBK cells were cultured in a monolayer and inoculated with BPIV3. At different time points after inoculation, the cell-virus suspension was harvested at $6,12,18,24,36,48$, and $60 \mathrm{~h}$, and $\operatorname{TCID}_{50}$ was measured. The growth curve of BPIV3 was plotted according to the results of $\mathrm{TCID}_{50}$. The results showed that BPIV3 proliferated rapidly from 24 to $36 \mathrm{~h}$ after infection, indicating active intracellular replication of the virus.

Because CPE is caused by intercellular fusion after BPIV3 invades the target cells, MDBK cells were inoculated with BPIV3 at the dose of 1 multiplicity of infection (MOI), and CPE was observed at different time points after infection. The results showed that lesions began to appear 

conditions combined with virus proliferation, cells infected at $24 \mathrm{~h}$ were used as the time point for proteomics analysis.

B

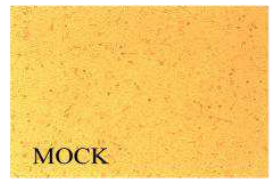

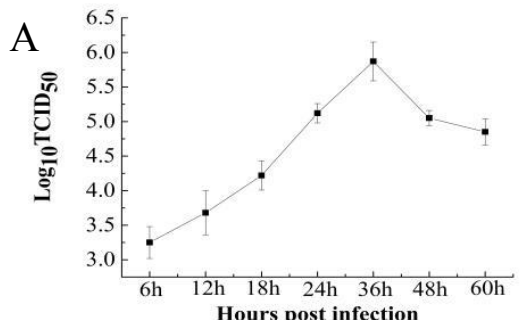

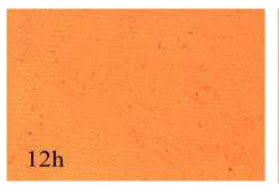

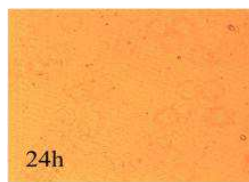

$36 \mathrm{~h}$

Fig. 1 Kinetic analysis of BPIV3 replication in MDBK cells (A) The growth curve of BPIV3; (B)

The cytopathic effect of BPIV3.

\section{Protein profiling and iTRAQ quantification}

The collected protein samples of BPIV3-infected and mock-infected MDBK cells were labeled with ITRAQ reagent in three biological repetitions. The quantitative information of the two experimental group ratios (ratio [infection/control]) was obtained by integrating the peptide segment information of three biological duplicates in the mock-infected group (control) and the BPIV3-infected group (infection).

The changes in the protein expression level between the two groups were analyzed based on statistical significance. A total of 2804 proteins were detected and quantified by LC-MS/MS. Among these proteins, 74 proteins were significantly upregulated and 42 proteins were markedly downregulated according to the change ratio of $\geq 1.5$ for the proteins and significant differences at $P<0.05$ (Fig. 2). The most significantly upregulated protein among the DEPs was vesicle-related membrane protein, which is related to autophagy. The most significantly downregulated protein was the integrin complement protein, which is a receptor protein of viral infection.

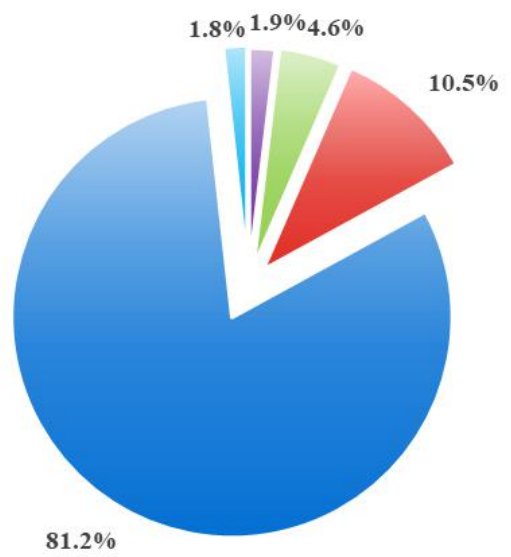

$-\mathrm{p}<0.001$

$=0.001<\mathrm{p}<0.01$

$=0.01<p<0.05$

- $\mathrm{p}>0.05$

- Unquantified proteins 
Fig. 2 The quantitation and significance of the 2804 identified proteins from BPIV3-infected and mock-infected groups.

GO annotations of the DEPs

The results of the GO enrichment analysis of the biological process showed that the DEPs were significantly enriched in five processes. These processes included single organism process, response to a stimulus, metabolic process, cell process, and biological regulation. The proteins involved in the biological regulation process were found most, followed by those involved in the stimulation response process. In this study, the proteins involved in the stimulation response process mainly included tyrosine phosphatase, signal transduction protein 1 , Rab5 GDP/GTP conversion factor 1 , interleukin-13 (IL-13), mitogen-activated protein kinase 7 (MAPK7), FOX transcription inhibitory factor 3(Foxp3), calcium phosphate, protein tyrosine phosphatase protein receptor, MAP3K10, human telomerase reverse transcriptase, and SSNA1. IL-13 is the most important inflammatory factor that causes airway inflammation. It plays a key role in the occurrence of chronic airway inflammatory disease, which induces high secretion of mucus. Foxp3 is a member of the Fox transcription factor family that plays an important role in maintaining the immune function of the body [19]. The DEPs in BPIV3-infected MDBK cells may cause the initial cellular stress response. The precise role of these DEPs in the BPIV3 infection process need to be further investigated.

A

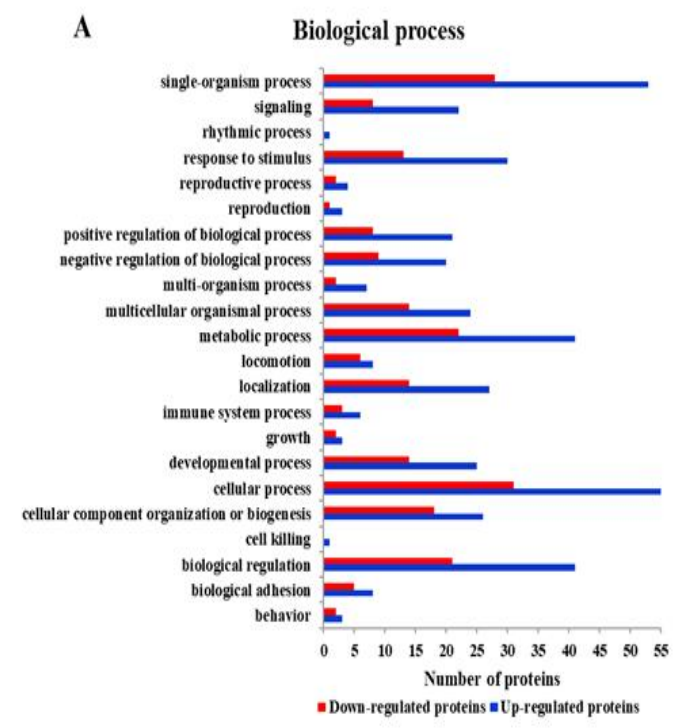

B

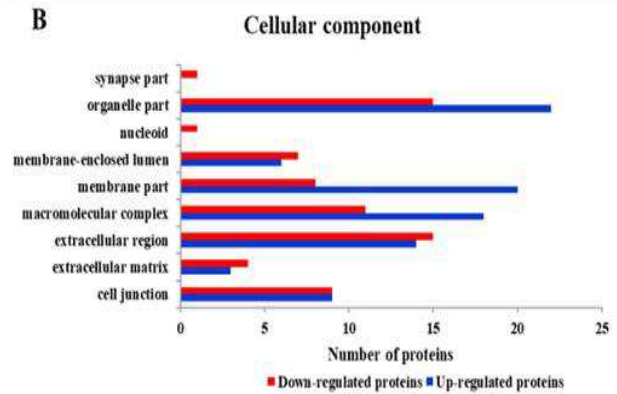

C

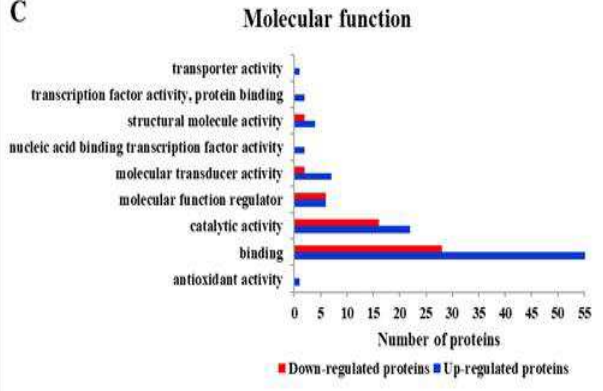

Fig. 3 The Gene Ontology (GO) categories of the differentially expressed proteins. (A) Biological process GO categories; (B) cellular component GO categories; (C)molecular functions GO categories. 
The KEGG pathway database is a collection map based on the molecular interaction pathways and cellular response networks. The DEPs were identified and mapped to six KEGG pathways, including metabolism, cellular processes, organismal systems, environmental information process, genetic information process, and disease pathways. The organismal systems and disease pathways were enrichment pathways, represented by 37 and 43 pathway groups, respectively.

A $\quad$ Down-regulated proteins $=$ Up-regulated proteins

Drug metabolism - cytochrome $\mathrm{P} 450$

Various types of $\mathrm{N}$-glycan biosynthesis Lysine degrad ation Inositol phosphate metabolism Galactose metabolism

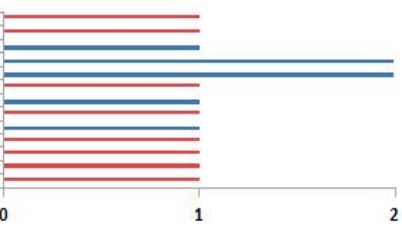

Number of proteins MAPK signaling pathway Ras signaling pathway

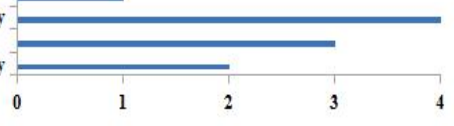

Number of proteins

E $\quad$ Down-regulated proteins $\square$ Up-regulated proteins

Inflammatory mediator regulation of TRP. Neurotrophin signaling pathway Serotonergic synapse GABAergic synaps Protein digestion and absorption Cardiac muscle contraction Oxytocin signaling pathway Insulin signaling pathway Fc gamma R-mediated phagocỵtosis Th17 cell differentiation Antigen processing and presentation Toll-like receptor signaling pathway Hematopoietic cell lineage -

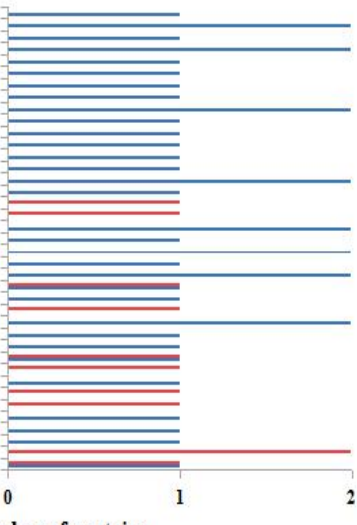

Number of proteins processes; (E) organismal systems; (F) diseases.
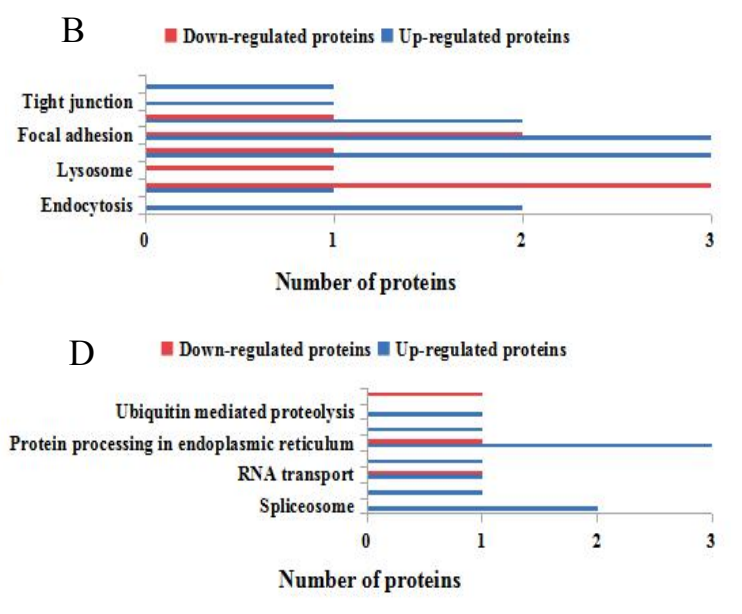

F

| Down-regulated proteins 1 Up-regulated proteins
Platinum drug resistance
Toxoplasmosis Toxoplasmosis
virus infection Hepatitis C HTLV-I infection Tuberculosis Legionellosis

Salmonella infection Vibrio cholerae infection Insulin resistance Viral myocarditis Arrhỵthmogenic right ventricular... Prion diseases

Amyotrophic lateral sclerosis (ALS) Allograft rejection

Autoimmune thyroid disease

Systemic lupus erỵthematosus Small cell lung cancer

Proteoglycans in cancer Transcription al misregulation in cancers Pathways in cancer Number of proteins

Fig.4 Analysis of the KEGG pathway of the differentially expressed proteins. (A) genetic information processing (B) Metabolism; (C) environmental information processing; (D) cellular

In the metabolic pathways, the DEPs participated in 13 pathways related to the metabolism of glucose, lipid, amino acid, and nucleotides (Fig 4-A). These pathways affect the metabolism of three major nutrients in cells. The cellular processes involved ten pathways (Fig.4-B), including 
the Focal adhesion pathway and the Phagosome pathway, both of which are involved in the viral infection process; the integrin protein is the key protein in these two pathways. The lysosome pathway, phagosome pathway, and autophagy are involved in the autophagy process of virus infection. The annotated proteins in the category of genetic information processing play a role in the synthesis, transport, proteolysis, and spliceosome of cells (Fig.4-D). The annotated proteins in the organismal systems category were related to antigen processing and presentation, NOD-like receptor signaling, Toll-like receptor signaling, complement and coagulation cascades, and Th1 and Th2 cell differentiation pathway groups. These pathways were primarily related to the immune response of the host to virus infection (Fig.4-E). The DEPs annotated in the disease category are shown in Fig.4-F. There are ten pathways associated with infectious diseases, five of which are associated with viral infections.

According to the profiling of DEPs, a relatively large number of proteins matched with the MAPK signaling pathway, including FGF13, ERK5, and MKK3. The KEGG pathway analysis revealed that MKK3 is involved in 14 pathways, indicating that MKK3 is a key regulatory protein during BPIV3 infection of MDBK cells (Fig. 4-C).

\section{Validation of the selected proteins by real-time quantitative PCR (qRT-PCR)}

To verify the DEPs identified by iTRAQ, the transcriptional levels of eight proteins were measured by qRT-PCR. In this study, eight proteins were randomly selected for qRT-PCR. The four upregulated proteins included AP-2 complex subunit beta protein (AP-2), FGF13, myristoylated alanine-rich C-kinase substrate (MARCS), and MKK3 proteins. The four downregulated proteins included MHC class II (MHCII), glutathione S-transferase (GSTA1), selenium protein $\mathrm{P}$ (SepP), and tissue factor pathway inhibitor (TFPI). As shown in Fig.5, the expression levels of these genes were consistent with the iTRAQ results. The results of qRT-PCR further verified the reliability of the ITRAQ experiment.
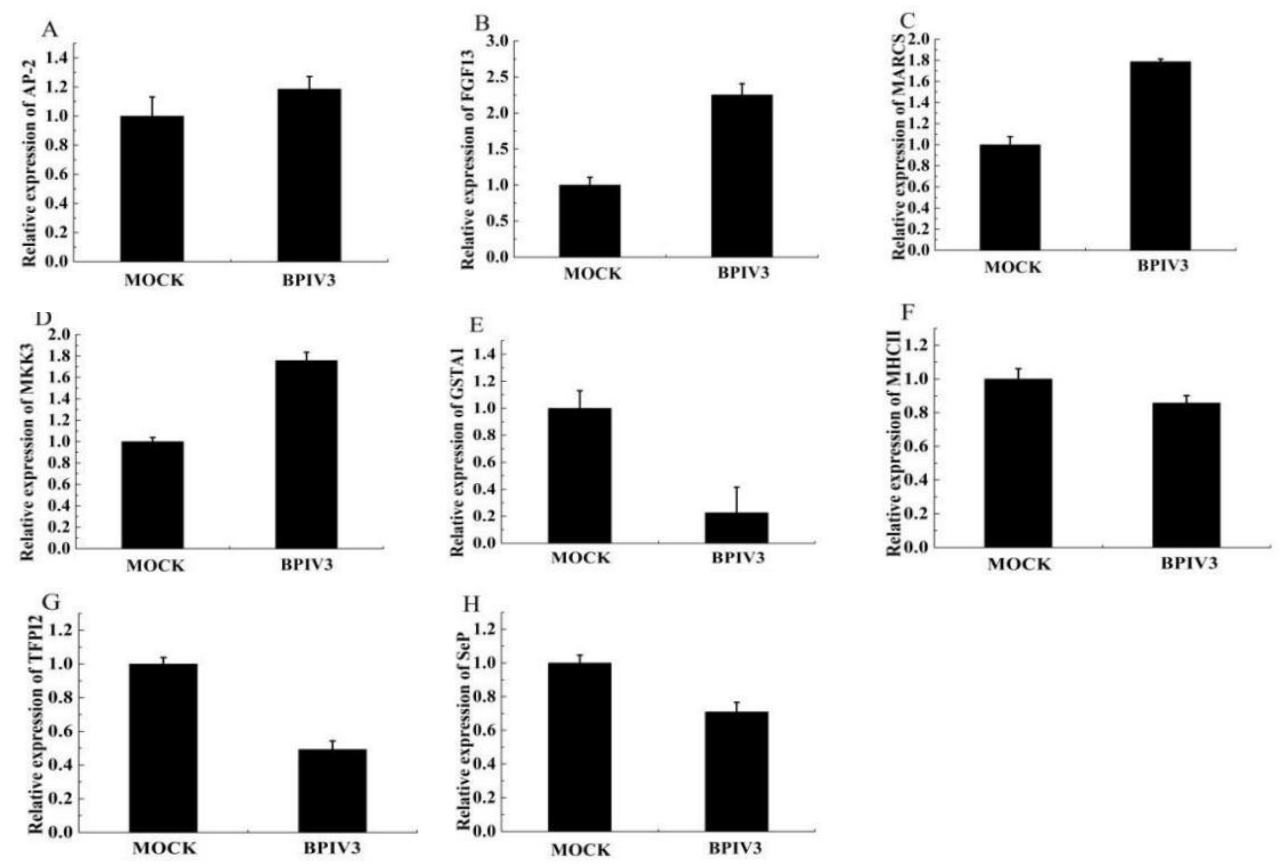
Fig.5 Expression profiles of the 8 differential expression genes by qRT-PCR. (A).Relative expression of AP-2; (B) Relative expression of FGF13; (C) Relative expression of MARCS; (D) Relative expression of MKK3; (E) Relative expression of GSTA1; (F) Relative expression of

(H) Relative expression of SepP.

\section{The effect of the p38 MAPK pathway on BPIV3 replication}

\section{BPIV3 infection activates the p38 MAPK pathway}

The MAPK pathway plays various roles in intracellular signaling network. MKK3 and MKK6 are recognized as upstream kinases of $\mathrm{p} 38$. The results of proteomics analysis showed that the MKK3 level was significantly upregulated after BPIV3 infection (Fig. 4C). Virus infection is considered as an extracellular stimulant that can activate p38 MAPK pathway[20-21]. It should be investigated whether BPIV3 infection activated the p38 MAPK pathway after MKK3 activation.

The expression of MKK3, p38, and phospho-p38 in BPIV3-infected cells was detected by western blotting assay. Cell samples were collected at 6, 12, and $24 \mathrm{~h}$ after BPIV3 infection. Compared to the mock group, the MKK3 expression levels were increased at different infection time points in the infected group. No change was observed in the p38 protein expression level, while the phospho-p38 expression level was significantly higher in the infected group than in the mock group at 12 and $24 \mathrm{~h}$ after BPIV3 infection (Fig. 6). Thus, BPIV3 infection induced MKK3 activation and p38 phosphorylation. The MKK3 expression level was consistent with previous proteomics results, which further verified the reliability of proteomics analysis.

A

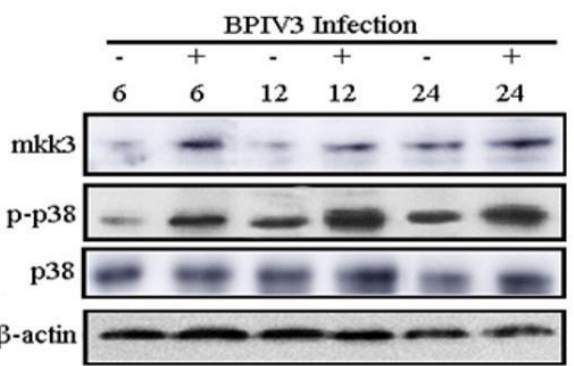

372

373

C

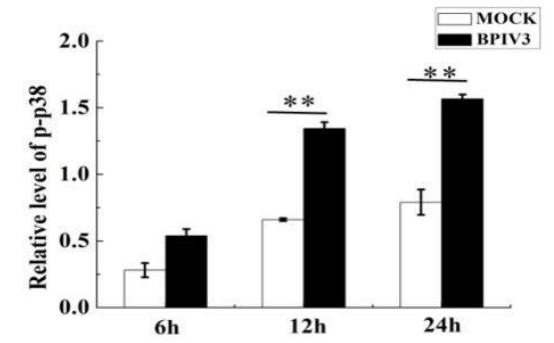

B

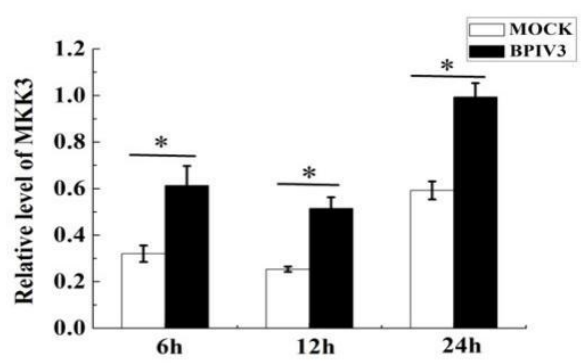

D

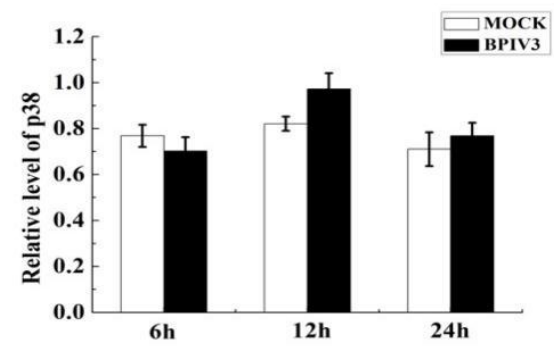

Fig.6 Effect of the BPIV3 infection on the protein expression in p38 MAPK pathway. (A) The protein expression in p38 MAPK pathway by Western blot; (B) Expression of MKK3; (C) Expression of p-p38; (D) Expres sion of p38. *(P<0.05), ** $(\mathrm{P}<0.01)$ 


\section{The effect of inhibiting p38 MAPK activation on BPIV3 replication}

To investigate whether the activation of the p38 MAPK pathway promotes BPIV3 proliferation, the cells were treated with SB202190, an inhibitor of the p38 MAPK pathway, $1 \mathrm{~h}$ before infection. MDBK cells were treated with SB202190 at 1.25, 5, and $10 \mathrm{M}$ concentrations. Cell samples were collected at $24 \mathrm{~h}$ after infection (MOI $=1)$.

The results are shown in Fig. 7. BPIV3 infection induced the phosphorylation of p38. After treatment with the inhibitor SB202190, the expression level of p38 was significantly decreased in a dose-dependent manner, indicating that the phosphorylation of p38 was inhibited by SB202190(Fig.7-A). The immunofluorescence analysis (IFA) results showed that BPIV3 replication could be inhibited by SB202190 in a dose-dependent manner (Fig. 7-B). The results are shown in Fig. 7C. The BPIV3 virus titer decreased by $1.8 \operatorname{logTCID}{ }_{50} / \mathrm{mL}$ after treatment with 10 M SB202190, indicating that the p38 MAPK pathway participates in the replication of BPIV3. The results showed that SB202190 could inhibit the proliferation of BPIV3. Thus, BPIV3 activated the p38 MAPK signaling pathway that is involved in its replication.
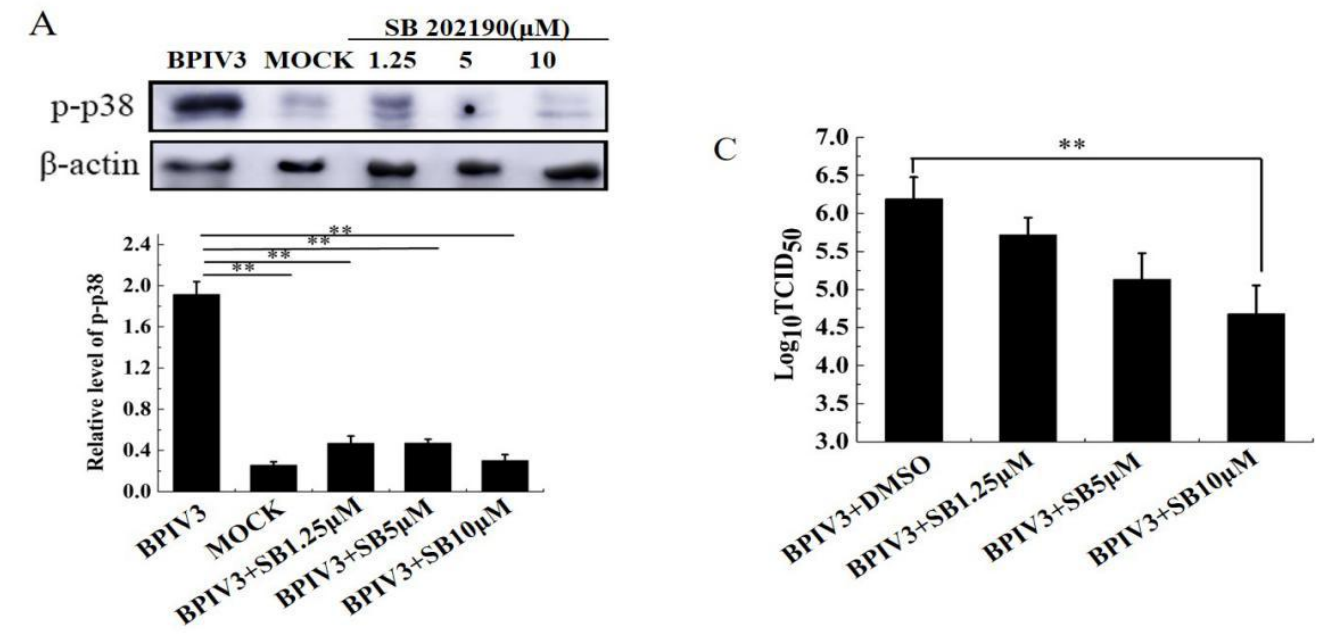

B

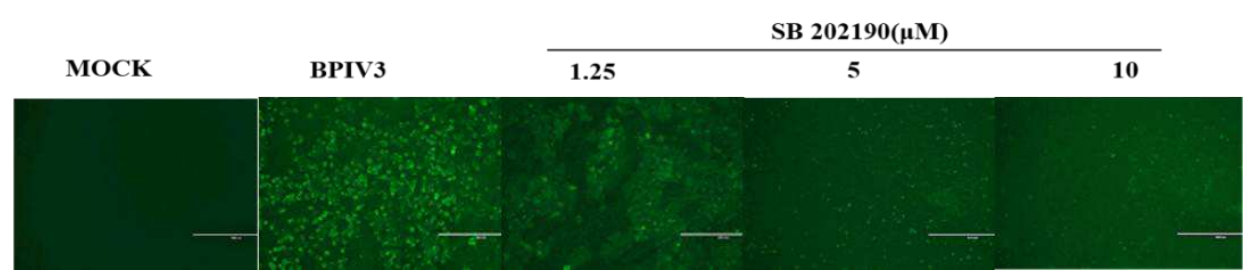

Fig.7 Effect of SB202190 on Bpiv3 replication(A) SB202190 impact on p38MAPK phosphorylation; (B) SB202190 impact on Bpiv3 infection ( $\times 100)$;(C)SB202190 impact on Bpiv3 $\mathrm{TCID}_{50} * *(\mathrm{P}<0.01)$

\section{Discussion}

iTRAQ LC-MS/MS is a powerful analytical tool for quantitative proteomics analysis that has 
been widely used in many studies [22-25]. Gray et.al used 2D gel electrophoresis proteomic to investigate in vitro cellular responses during BPIV3 infection[26]. In the present study, we first applied the iTRAQ LC-MS/MS approach to determine the profiles of DEPs in MDBK cells infected with BPIV3 at various time points of infection. A total of 116 DEPs were identified at $24 \mathrm{~h}$ after infection based on a fold change of $\geq 1.5$ and p-value $<0.05$ (data not show). On the basis of GO analysis, the DEPs were classified into 19, 11, and 9 categories for biological processes, cellular components, and molecular functions, respectively (Fig. 3). The pathway analysis identified the pathways based on the number of DEPs (Fig. 4). These data could provide a basis for understanding the pathogenetic mechanisms of BPIV3 infection.

The results showed that the PI3K-Akt signaling pathway and the MAPK signaling pathway play important roles in the progression of BPIV3 infection. According to the profiles of DEPs in these two signaling pathways, only ITGB3 was downregulated, while the remaining proteins were upregulated. The analysis of the DEPs in these pathways showed that the number of matched proteins in the MAPK signaling pathway was relatively large, including FGF13, ERK5, and MKK3. The KEGG pathway results indicated that MKK3 was involved in 14 pathways, which suggested that MKK3 is a key regulatory protein during BPIV3 infection. Previous studies have shown that the MAPK signaling pathway is a target of respiratory viruses, which regulates various stages of the infection process [27-28].

The MAPK cascade plays various roles in intracellular signaling network pathways. MKK3 and MKK6 are recognized as upstream kinases of p38 that can directly phosphorylate tyrosine and serine/threonine residues to activate p38 [29]. Viral infection is thought to be an extracellular stimulant that activates this pathway. In infectious salmon anemia virus (ISAV) infection, immunohistochemical detection showed that the phosphorylation level of p-ERk1/p-p38 in the lungs of sheep infected with JSRV was significantly increased compared to that in healthy sheep[30]. In HBV-infected HuH-7 cells, the results showed that HBV replication activated JNK and p38[31]. In our proteomics study, the MKK3 level was significantly upregulated $24 \mathrm{~h}$ after BPIV3 infection as compared to that in the control group. Therefore, we detected the expression level of the p38 MAPK pathway proteins after BPIV3 infection.

First, we investigated whether BPIV3 infection activates the p38 MAPK pathway. The results showed that BPIV3 induces the phosphorylation of p38 after infection. Compared to the control group, the phosphorylated p38 expression was significantly increased after $6 \mathrm{~h}$ of BPIV3 infection, demonstrating that BPIV3 can induce the activation of the p38 MAPK pathway in the early stage of infection.

Multiple extracellular stresses activate the MKK3-p38 MAPK cascade, including specific antigens, proinflammatory cytokines, ultraviolet light, heat shock, and other stress responses[32]. In accordance with the results of the mechanism of Coxsackie virus activation of p38 MAPK, we hypothesized that in the early stage of infection[33], the binding of BPIV3 HN protein to the receptor on the surface of the cell membrane induced membrane fusion, and the cascade reaction 
This project was supported by the Heilongjiang Bayi Agricultural University natural science talent of MKK3-p38 MAPK was temporarily activated. The progression of BPIV3 infection was gradually prolonged, and the phosphorylation of p38 MAPK was significantly increased $24 \mathrm{~h}$ after infection. In the late stage of infection, p38 was still continuously activated, which was speculated to be due to the release of proinflammatory cytokines induced by BPIV3 infection; the release of proinflammatory cytokines into the extracellular domain bound to the receptor further enhanced the activation of the p38 MAPK pathway[34-35].

Many studies have shown that $\mathrm{p} 38$ is required for the replication of viruses. The activation of the MAPK pathways by viruses such as stimulates the JNK and p38 MAPK pathways to promote the release of virions [33]. In porcine reproductive and respiratory syndrome virus infection, the virus replication was inhibited after inhibition of the JNK and p38 pathways[36]. The same results were noted in PEDV infection [37]. To detect the role of the p38 MAPK pathway in BPIV3 replication, virus titer and CPE were analyzed. The results showed that the inhibitor SB202190 significantly inhibited virus replication in a dose-dependent manner. It was also found that p38 expression was inhibited after treatment with SB202190. Compared with the untreated group, virus titer was significantly decreased after treatment of the cells with the inhibitor. The inhibitor also showed a significant inhibitory effect on virus-induced CPE in a dose-dependent manner. These results revealed that the activation of the p38 MAPK pathway facilitated replication of BPIV3.

In this study, DEPs in BPIV3-infected MDBK cells were identified and quantitatively analyzed by iTRAQ and LC-MS-based proteomics analysis. Most of the DEPs were proteins related to inflammatory response, immune response, and lipid metabolism. Although many significantly up- or downregulated proteins and pathways are closely related to the symptoms or pathological responses to BPIV3 infection, further functional investigations are required to understand the pathogenic mechanisms and molecular responses of host cells to BPIV3 infection.

The results of the present study indicated that BPIV3 infection activates the p38 MAPK pathway, which is essential for its replication. Proteomics and western blot analyses showed that BPIV3 infection activated the p38 MAPK signaling pathway. Our future research will focus on which step of virus replication is affected by $\mathrm{p} 38$ activation.

\section{Conflicts of interest}

None. 
projects (HKKYZD190305), Heilongjiang Bayi Agricultural University Doctor's Research Foundation, (XDB201816), Heilongjiang Bayi Agricultural University Scientific Research Team Support project (TDJH201904). We thank International Science Editing ( http://www.internationalscienceediting.com ) for editing this manuscript.

\section{References}

1.Kale, M. , Ozturk, D. , Hasircioglu, S. , Pehlivanoglu, F. , \& Turutoglu, H. . (2013). Some viral and bacterial respiratory tract infections of dairy cattle during the summer season. Acta Veterinaria, 63(2-3), 227-236.

2.Kirchhoff, J., Uhlenbruck, S. , Goris, K. , Keil, Günther M, \& Herrler, G. . (2014). Three viruses of the bovine respiratory disease complex apply different strategies to initiate infection. Veterinary Research, 45(1), 20.

3.Grissett, G. P. , White, B. J. , \& Larson, R. L. . (2015). Structured literature review of responses of cattle to viral and bacterial pathogens causing bovine respiratory disease complex. Journal of Veterinary Internal Medicine, 29(3), 770-780.

4.Ellis, J. A. . (2010). Bovine parainfluenza-3 virus. Veterinary Clinics of North America: Food Animal Practice, 26(3), 575-593.

5.Henrickson K J(2011). Parainfluenza Viruses. John Wiley \& Sons, Ltd， 242-264.

6. Durbin, A. P. , Mcauliffe, J. M. , Collins, P. L. , \& Murphy, B. R. . (1999). Mutations in the c, d, and $\mathrm{v}$ open reading frames of human parainfluenza virus type 3 attenuate replication in rodents and primates. Virology, 261(2), 319-330.

7.Bousse, T. , \& Takimoto, T. . (2006). Mutation at residue 523 creates a second receptor binding site on human parainfluenza virus type 1 hemagglutinin-neuraminidase protein. Journal of Virology, 80(18), 9009.

8.Jardetzky, T. S. , \& Lamb, R. A. . (2014). Activation of paramyxovirus membrane fusion and virus entry. Current Opinion in Virology, 5, 24-33.

9.Durbin, A. P. , Mcauliffe, J. M. , Collins, P. L. , \& Murphy, B. R. . (1999). Mutations in the c, d, and $\mathrm{v}$ open reading frames of human parainfluenza virus type 3 attenuate replication in rodents and primates. Virology, 261(2), 319-330. 
10. Gisa, Gerold, Janina, Bruening, Thomas, \& Pietschmann. (2016). Decoding protein networks during virus entry by quantitative proteomics. Virus Research. 11.Seggerson K, Tang L, Moss EG (2002). Two genetic circuits repress the Caenorhabditis elegans heterochronic gene lin-28 after translation initiation. Dev Biol 243:215-225. 12.Kang, An, Liurong, Fang, Rui, \& Luo, et al. (2014). Quantitative proteomic analysis reveals that transmissible gastroenteritis virus activates the jak-stat1 signaling pathway. Journal of Proteome Research, 13(12), 5376-5390. 13.Sun, D. , Shi, H., Guo, D. , Chen, J., Shi, D. , \& Zhu, Q. , et al. (2015). Analysis of protein expression changes of the vero e6 cells infected with classic pedv strain cv777 by using quantitative proteomic technique. Journal of Virological Methods, 218, 27-39.

14. Li, L. , Yu, L. , \& Hou, X. . (2017). Cholesterol-rich lipid rafts play a critical role in bovine parainfluenza virus type 3 (bpiv3) infection. Research in Veterinary Science, 114, 341-347. 15. Zhu, Y. M. , Shi, H. F. , Gao, Y. R. , Xin, J. Q. , Liu, N. H. , \& Xiang, W. H.. (2011). Isolation and genetic characterization of bovine parainfluenza virus type 3 from cattle in china. Veterinary Microbiology,149( 3-4), 446-451. 16.Chowdhury, S. I. , Coats, J., Neis, R. A. , Navarro, S. M. , Paulsen, D. B. , \& Feng, J. M. . 17.Yazici, Z. , Ozan, E. , Tamer, C. , Muftuoglu, B. , \& Albayrak, H. . (2020). Circulation of molecular characterization. Animals, 10(9), 1700. 18.A, X. G. , A, H. H. , A, F. C. , A, Z. L. , A, S. Y. , \& B, S. C. , et al. (2016). Itraq-based comparative proteomic analysis of vero cells infected with virulent and cv777 vaccine strain-like strains of porcine epidemic diarrhea virus - sciencedirect. Journal of Proteomics, 130, 65-75.

19.Khattri, R. , Cox, T. , Yasayko, S. A. , \& Ramsdell, F. . (2003). An essential role for scurfin in $\mathrm{cd} 4+\mathrm{cd} 25+\mathrm{t}$ regulatory cells. Nature Immunology, 4(4), 337-342.

20.Víctor, H., Olavarría, Pablo, Recabarren, \& Fernanda, et al. (2015). Isav infection promotes apoptosis of shk-1 cells through a ros/p38 mapk/bad signaling pathway. Molecular Immunology, 64(1), 1-8. 
21.Fu, Y., Yip, A., Seah, P. G. , Blasco, F. , Shi, P. Y. , \& Hervé, Maxime. (2014). Modulation of inflammation and pathology during dengue virus infection by p38 mapk inhibitor sb203580. Antiviral Research, 110, 151-157.

22.China, I. . (2015). Quantitative itraq lc-ms/ms proteomics reveals the proteome profiles of df-1 cells after infection with subgroup j avian leukosis virus. Biomed Res Int, 2015(3), 395307.

23.Lu, Q. , Bai, J. , Zhang, L. , Liu, J. , Jiang, Z. , \& Michal, J. J. , et al. (2013). Two-dimensional liquid chromatography-tandem mass spectrometry coupled with isobaric tags for relative and absolute quantification (itraq) labeling approach revealed first proteome profiles of pulmonary alveolar macrophages infected with porcine reproductive and respiratory syndrome virus. Journal of Proteomics, 79(5), 72-86.

24.Hu, F. , Li, Y. , Yu, K. , Ma, X. , \& Huang, B. . (2019). Proteome analysis of reticuloendotheliosis-virus-infected chicken embryo fibroblast cells through itraq-based quantitative proteomics. Archives of Virology, 164(12), 2995-3006.

25.Zhong, C. , Li, J., Mao, L. , Liu, M. , \& Liao, Z. . (2019). Proteomics analysis reveals heat shock proteins involved in caprine parainfluenza virus type 3 infection. BMC Veterinary Research, 15(1).

26.Gray, D. W. , Welsh, M. D. , Doherty, S. , \& Mooney, M. H. . (2017). Identification of candidate protein markers of bovine parainfluenza virus type 3 infection using an in vitro model. Veterinary Microbiology, 203, 257.

27.Wang, C. ,Wei, D, .Xu, M, .Zhang, .R. Liu, B, .\& Wang, G..(2014). The role of p38MAPK in acute lung injury induced by H9N2 influenza virus isolated from swine. Acta veterinaria et zootechnica sinica, 045(2), 281-288.

28. Lee, N. , Wong, C. K. , Chan, P. K. S. , Lun, S. W. M. , Lui, G. , \& Wong, B. . (2007). Hypercytokinemia and hyperactivation of phospho-p38 mitogen-activated protein kinase in severe human influenza a virus infection. Clinical Infectious Diseases(6), 723-731.

29. B, M. C. A. , B, J. O. , A, J. B. S. , \& A, O. H. N. . (2011). Map kinases in inflammatory bowel disease. Clinica Chimica Acta,412( 7-8), 513-520.

30.Víctor, H., Olavarría, Pablo, Recabarren, \& Fernanda, et al. (2015). Isav infection promotes apoptosis of shk-1 cells through a ros/p38 mapk/bad signaling pathway. Molecular Immunology, 64(1), 1-8. 
31.PU, C. ,ZHOU, H. , PENG, L. , SONG, Y. , \& FENG, Y. .(2011). Influences of Hepatitis B virus $\mathrm{X}$ protein on the expression of MKK3 and p38MAPK in hepatoma carcinoma cell. Modern Oncology19( 04): $0640-0644$.

32.Gautier, A. , Deiters, A. , \& Chin, J. W. . (2011). Light-activated kinases enable temporal dissection of signaling networks in living cells. Journal of the American Chemical Society, 133(7), 2124-2127.

33.Si, X. , Luo, H. , Morgan, A. , Zhang, J. , Wong, J. , \& Yuan, J. , et al. (2005). Stress-activated protein kinases are involved in coxsackievirus b3 viral progeny release. Journal of Virology,79(22), 13875.

34.Pettus, L. H. , \& Wurz, R. P. . (2008). Small molecule p38 map kinase inhibitors for the treatment of inflammatory diseases: novel structures and developments during 20062008. Current Topics.

35.Choi, M. S. , Heo, J. , Yi, C. M. , Ban, J. , Lee, N. J. , \& Lee, N. R. , et al. (2016). A novel p38 mitogen activated protein kinase (mapk) specific inhibitor suppresses respiratory syncytial virus and influenza a virus replication by inhibiting virus-induced p38 mapk activation. Biochemical \& Biophysical Research Communications, 477(3), 311-316.

36.Tong, A. , Jiang, L., Chia, S. , \& Dong Y..(2020). Molecular and Cellular Mechanisms for PRRSV Pathogenesis and Host Response to Infection. Virus Research, 286,1-11.

37. Lee, C. , Kim, Y. , \& Jeon, J. H. . (2016). Jnk and p38 mitogen-activated protein kinase pathways contribute to porcine epidemic diarrhea virus infection. Virus Research, 1-12. 


\section{Figures}
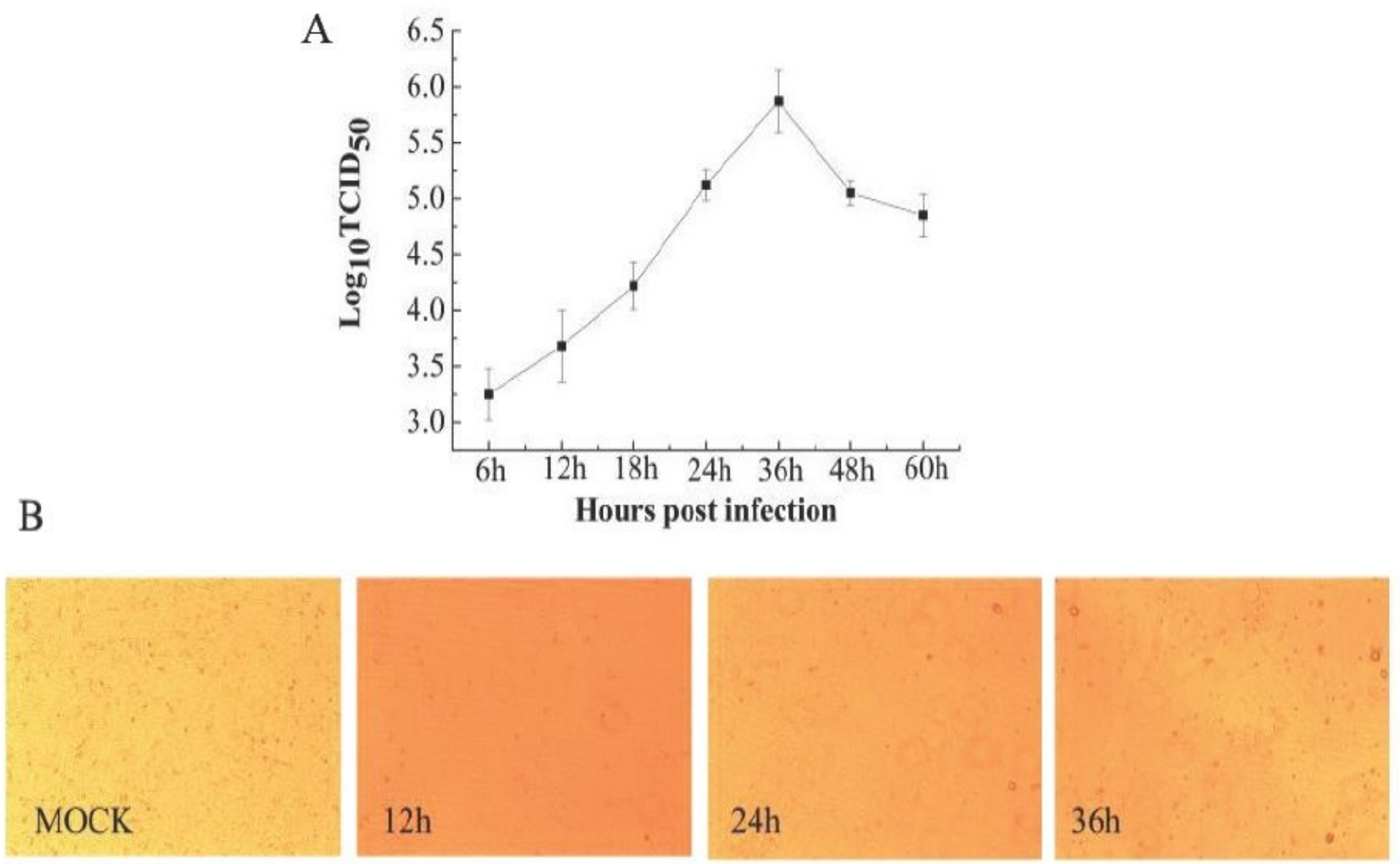

\section{Figure 1}

Kinetic analysis of BPIV3 replication in MDBK cells (A) The growth curve of BPIV3; (B) The cytopathic effect of BPIV3. 


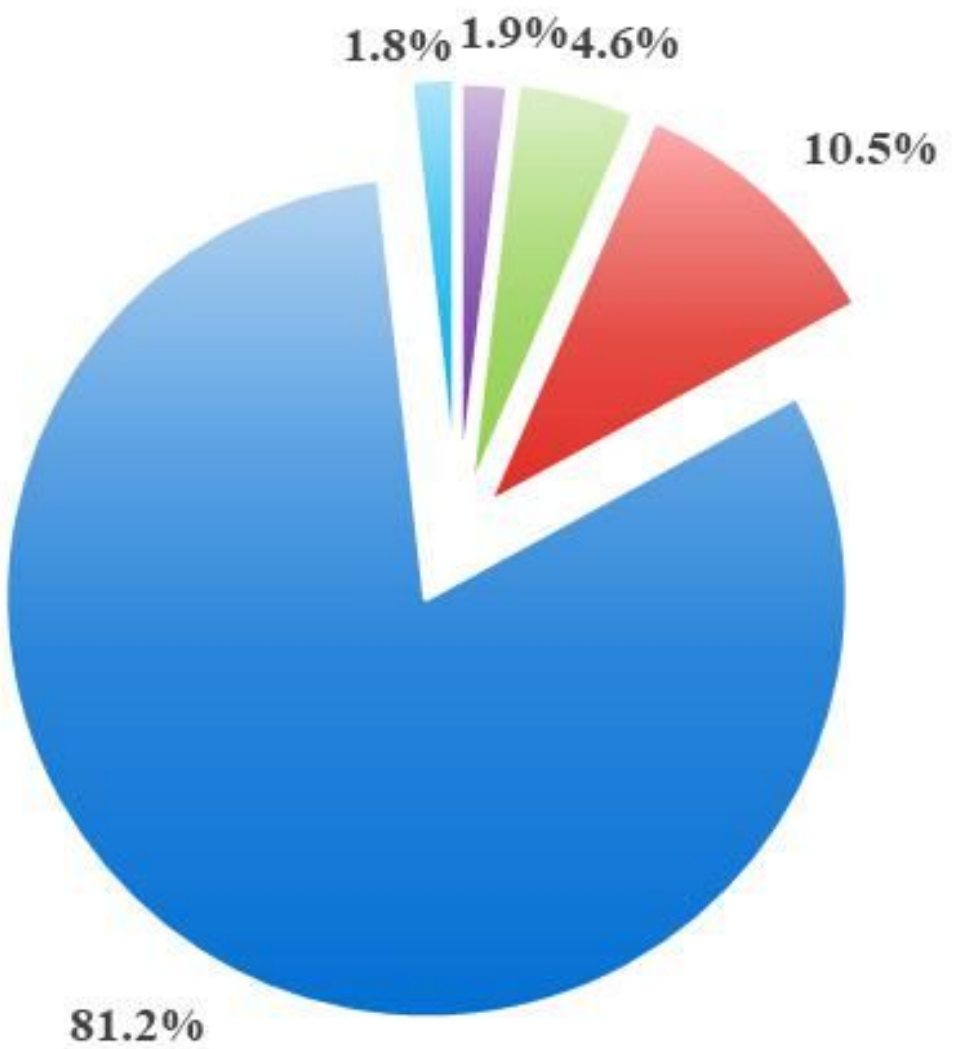

$\mathrm{p}<0.001$

$=0.001<\mathrm{p}<0.01$

$=0.01<p<0.05$

$-p>0.05$

- Unquantified proteins

\section{Figure 2}

The quantitation and significance of the 2804 identified proteins from BPIV3-infected and mock-infected groups.

A

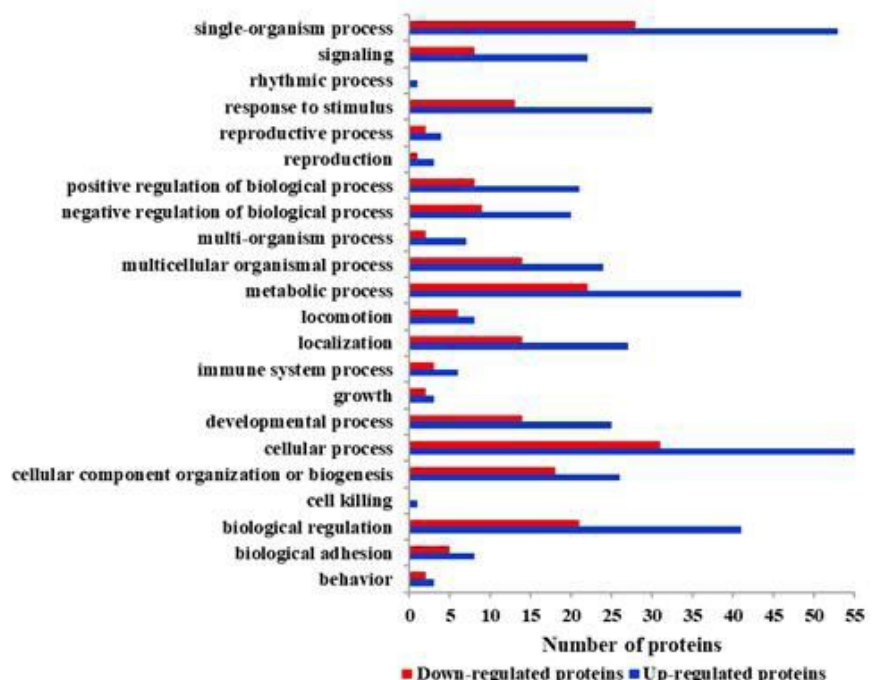

B Cellular component

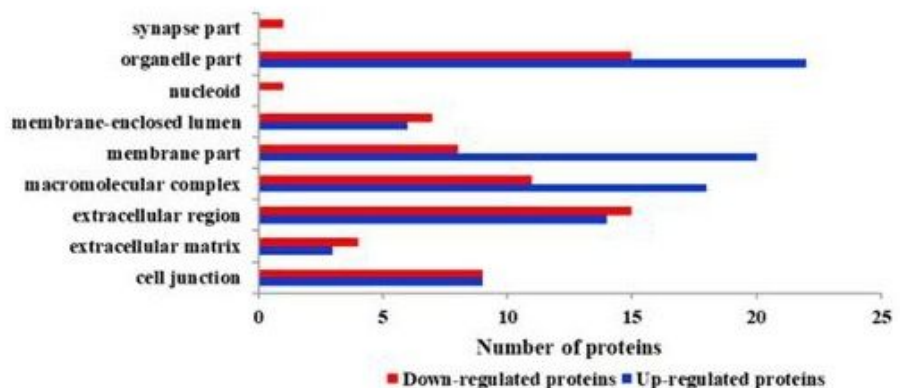

C

Molecular function

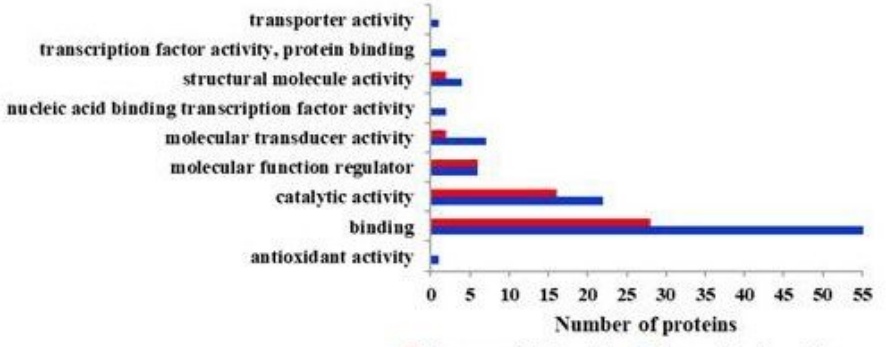

- Down-regulated proteins $\equiv$ Up-regulated proteins 


\section{Figure 3}

The Gene Ontology (GO) categories of the differentially expressed proteins. (A) Biological process GO categories; (B) cellular component GO categories; (C)molecular functions GO categories.
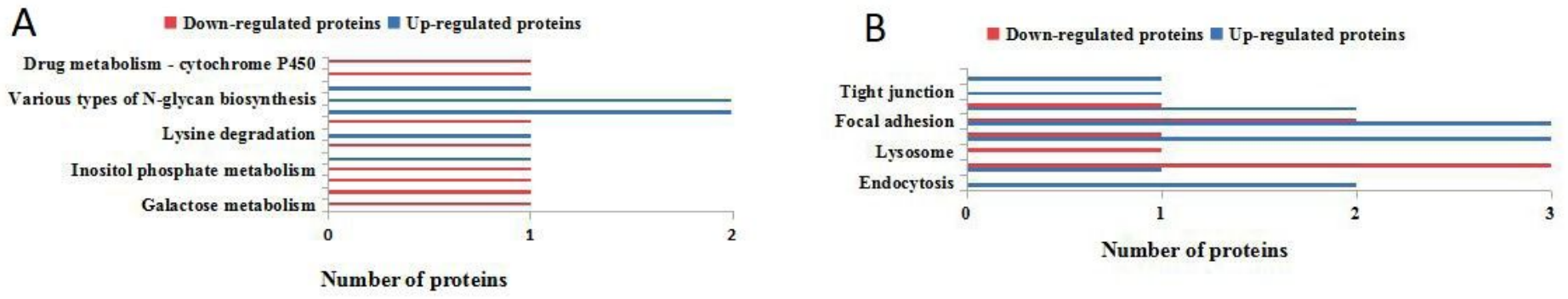

C

[ Down-regulated proteins $\mid$ Up-regulated proteins

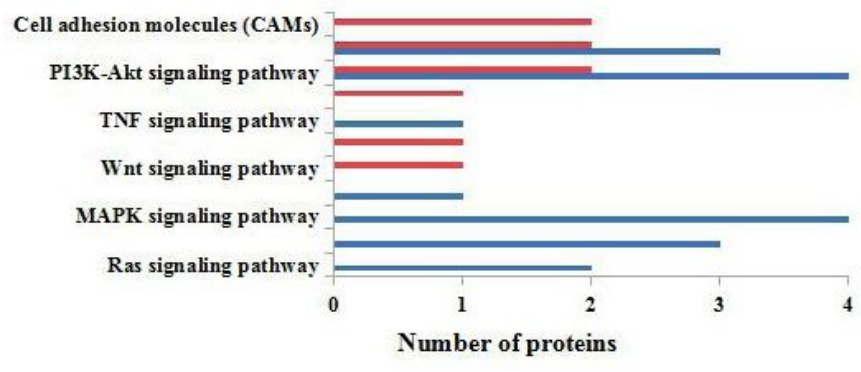

E

= Down-regulated proteins $\mid$ U p-regulated proteins

Inflammatory mediator regulation of TRP...

Neurotrophin signaling pathway

Serotonergic synapse

GABAergic synapse

Protein digestion and absorption

Cardiac muscle contraction

Oxytocin signaling pathway

Insulin signaling pathway

Fc gamma R-mediated phagocytosis

Th17 cell differentiation

Antigen processing and presentation

Toll-like receptor signaling pathway

Hematopoietic cell lineage

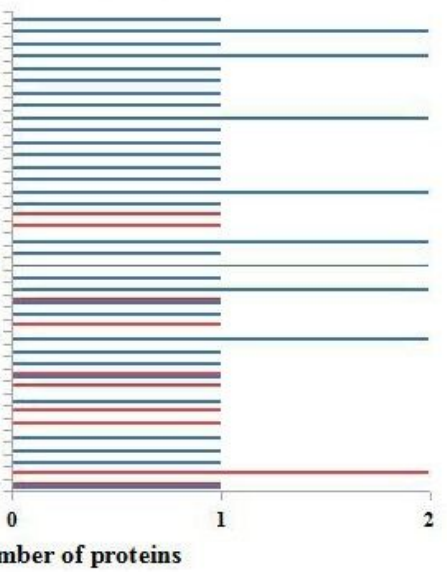

Number of proteins

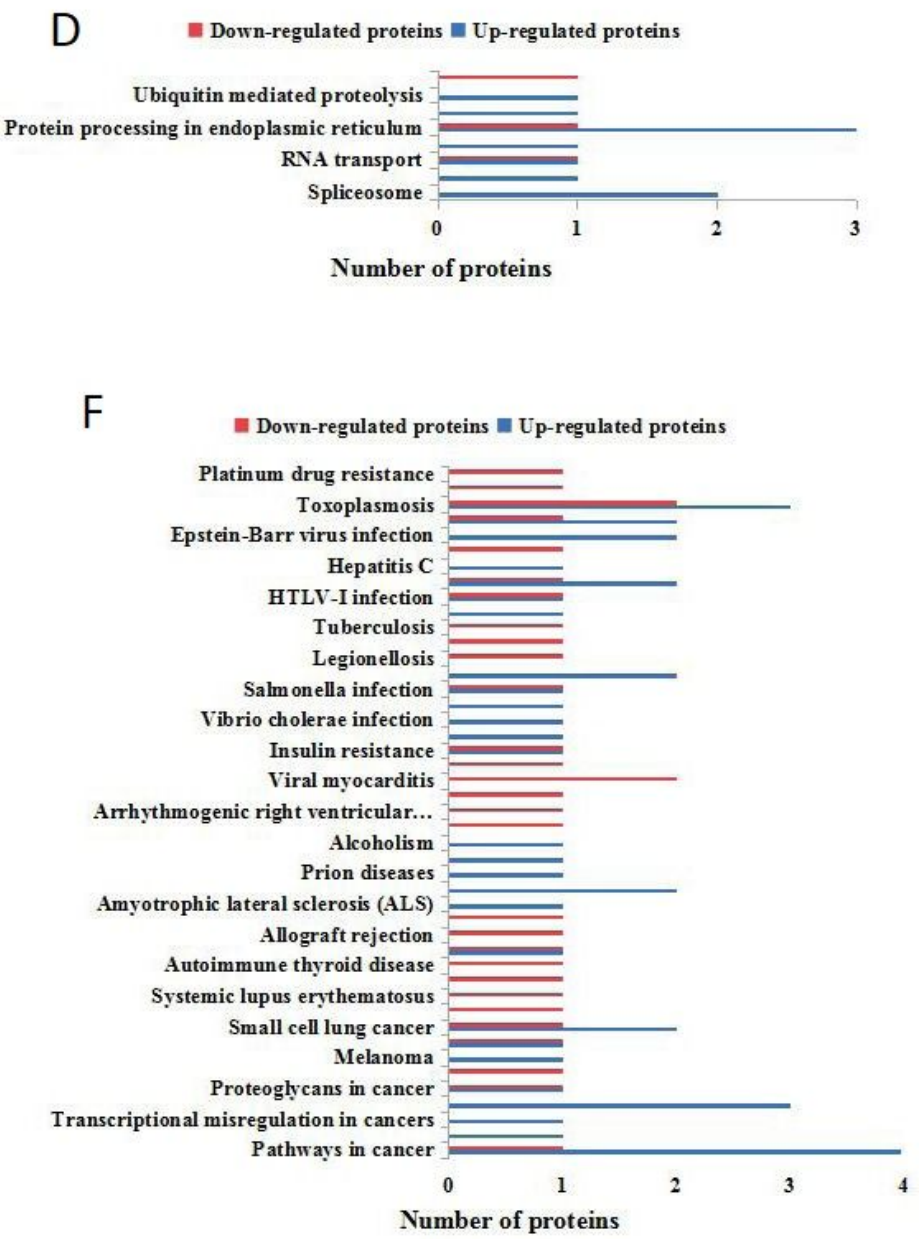

\section{Figure 4}

Analysis of the KEGG pathway of the differentially expressed proteins. (A) genetic information processing (B) Metabolism; (C) environmental information processing; (D) cellular processes; (E) organismal systems; (F) diseases. 

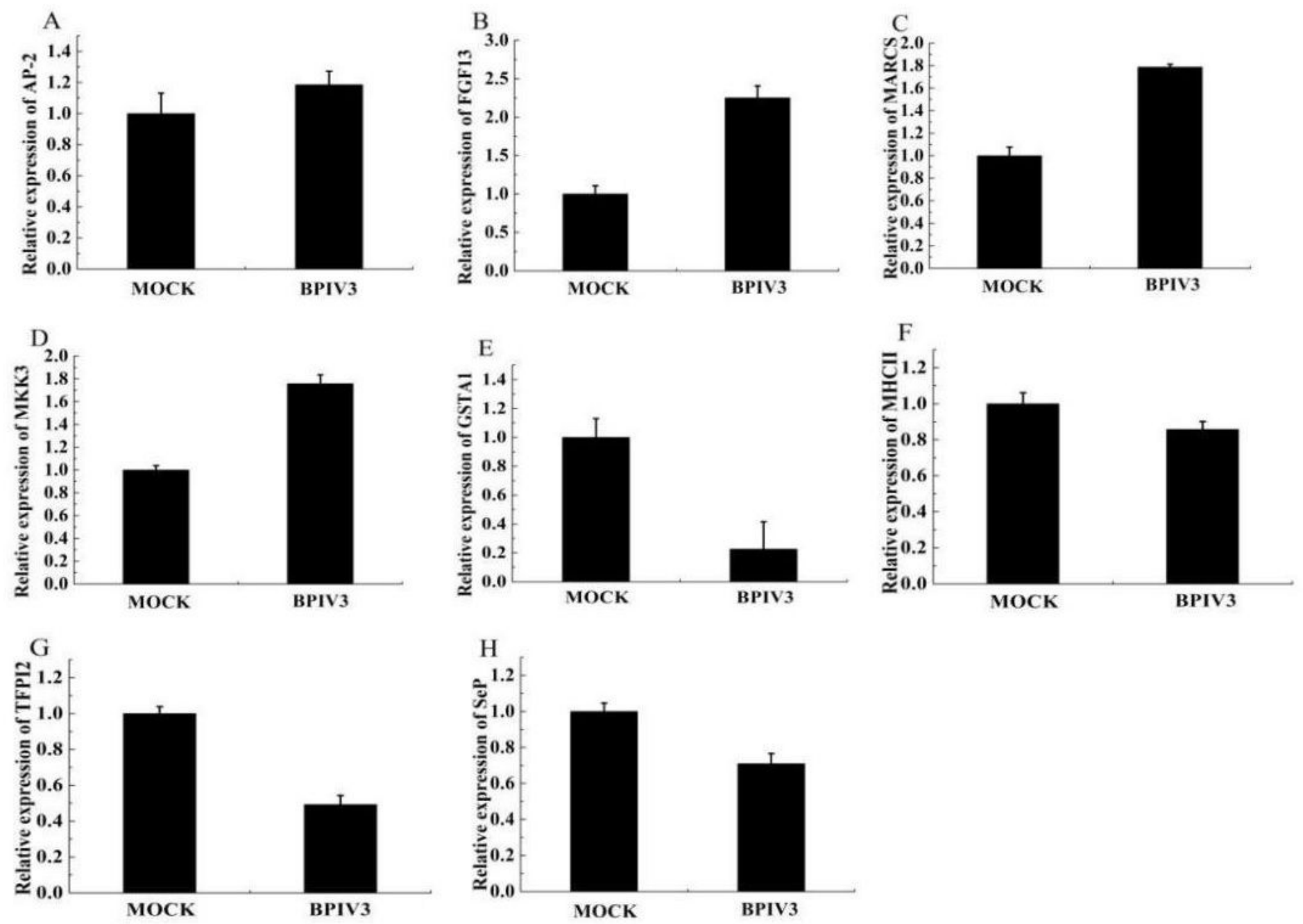

Figure 5

Expression profiles of the 8 differential expression genes by qRT-PCR. (A).Relative expression of AP-2; (B) Relative expression of FGF13; (C) Relative expression of MARCS; (D) Relative expression of MKK3; (E) Relative expression of GSTA1; (F) Relative expression of MHCII; (G) Relative expression of TFPI2; $(\mathrm{H})$ Relative expression of SepP. 
A

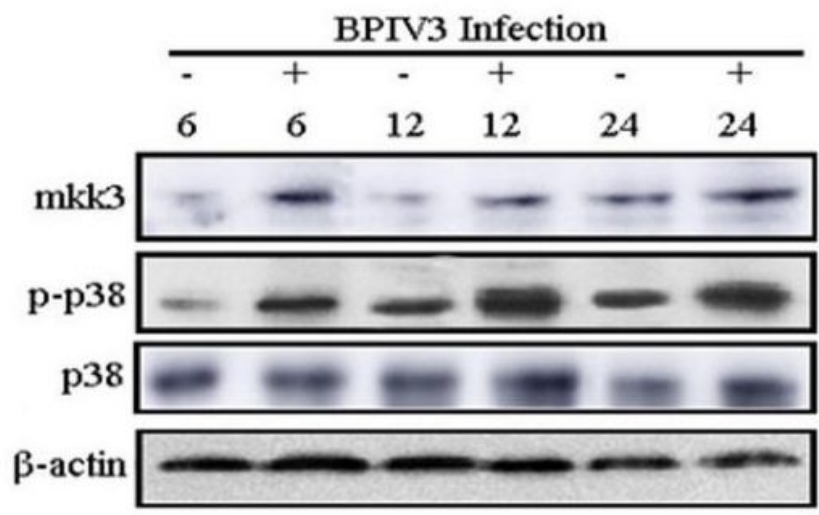

$\mathrm{C}$

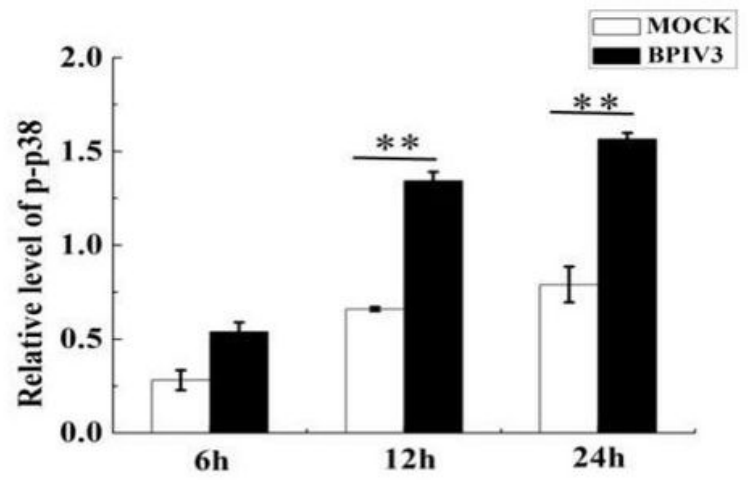

$\mathrm{B}$

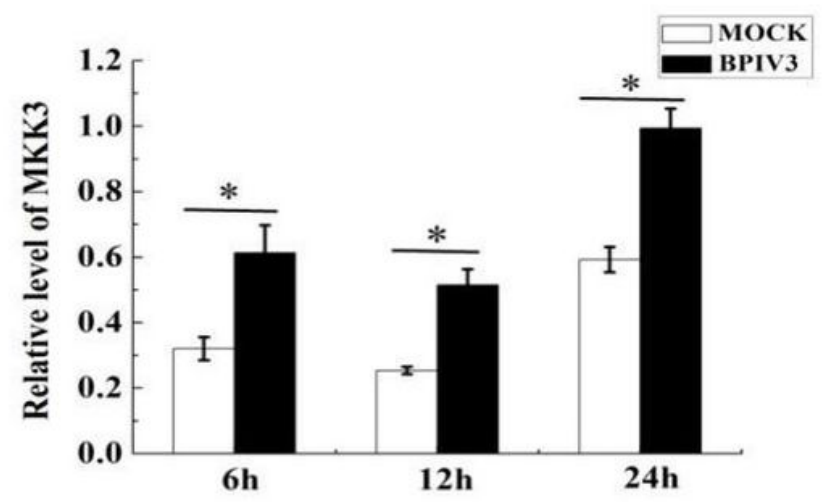

$\mathrm{D}$

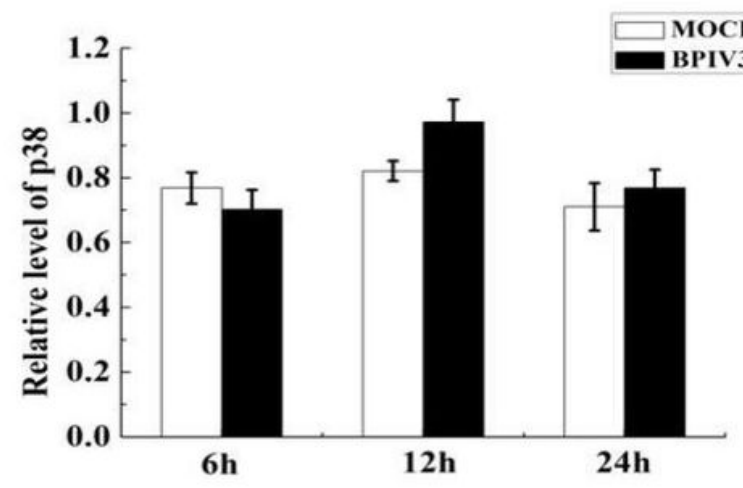

Figure 6

Effect of the BPIV3 infection on the protein expression in p38 MAPK pathway. (A) The protein expression in p38 MAPK pathway by Western blot; (B) Expression of MKK3; (C) Expression of p-p38; (D) Expression of $p 38 . *(P<0.05), * \star(P<0.01)$ 
A

SB $202190(\mu M)$

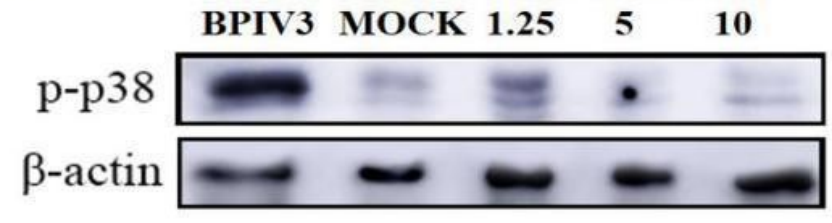

C
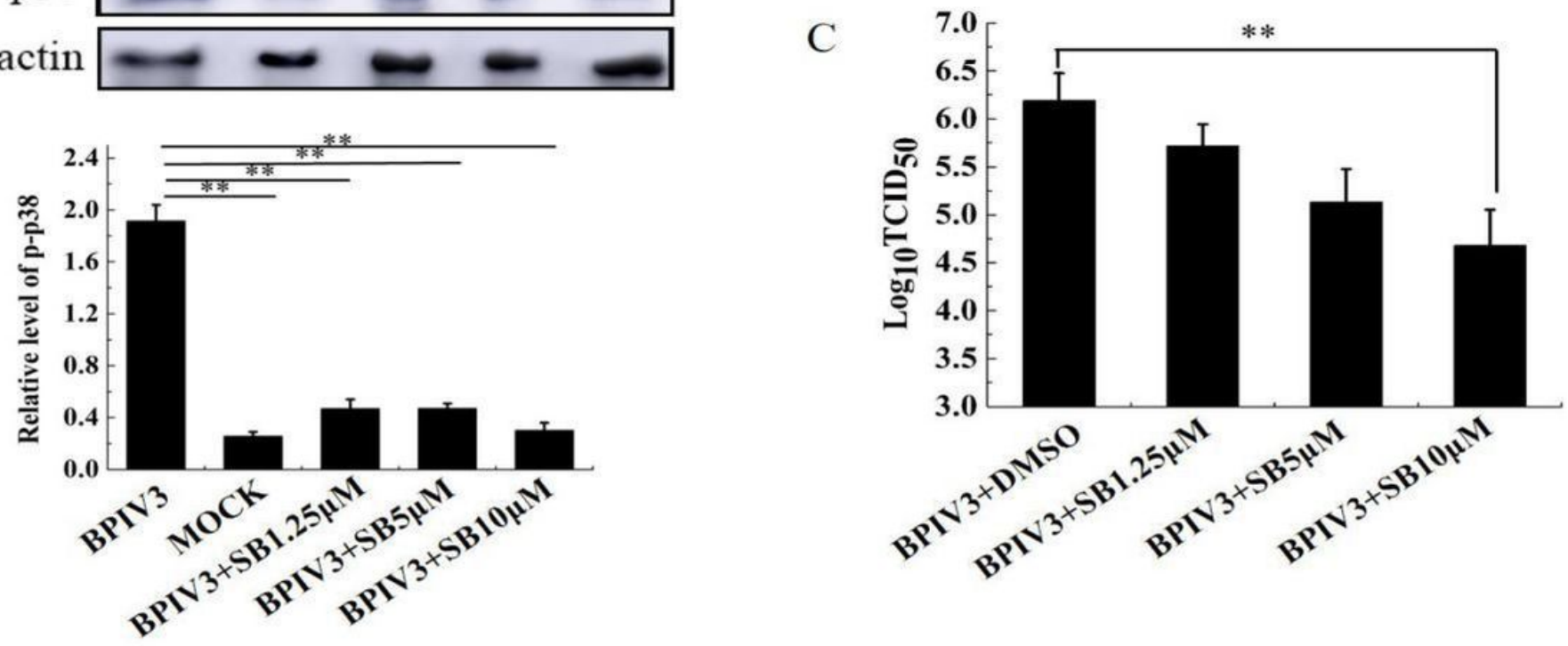

B

SB $202190(\mu M)$

MOCK

BPIV3

$\mathbf{1 . 2 5}$

10

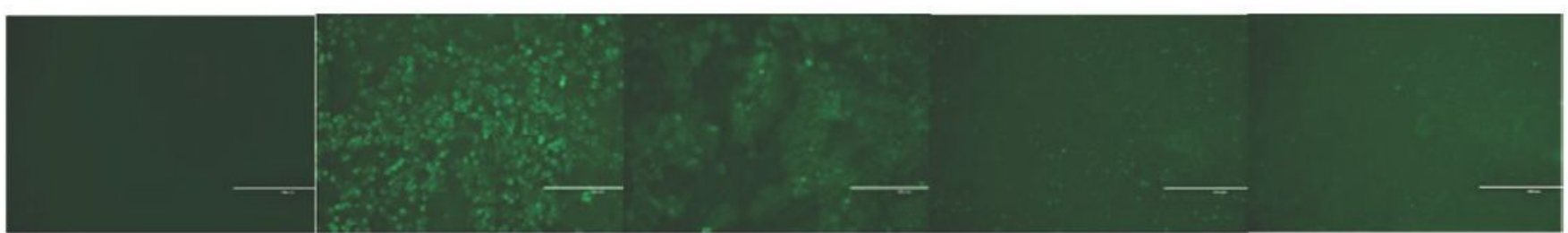

Figure 7

Effect of SB202190 on Bpiv3 replication(A) SB202190 impact on p38MAPK phosphorylation; (B) SB202190 impact on Bpiv3 infection (×100); (C)SB202190 impact on Bpiv3 TCID50.** $(P<0.01)$ 